\title{
PRINCIPLA IURIS: OPTIMISMO METODOLÓGICO Y REAFIRMACIÓN DE LA CULTURA DE LOS DERECHOS *
}

\author{
Tecla Mazzarese ** \\ Universidad de Brescia
}

RESUMEN. Principia iuris, de L. FerRAJoli, ofrece una teoría comprehensiva del Derecho y de la democracia de una importancia singular en la literatura internacional de las últimas décadas. Después de hacer referencia a algunas de sus principales características distintivas, este artículo pone el énfasis en la significación del aparato teórico y argumentativo de Principia iuris, que permite a quien esté preocupado por su protección, reaccionar y responder a la diaria y progresiva deslegitimación de la doctrina y la cultura de los derechos fundamentales. En especial, el artículo se centra en la significación de la teoría de FERRAJOLI para responder a cinco alegaciones generalizadas que pretenden socavar la doctrina y la cultura de los derechos fundamentales: a) el restablecimiento de la guerra como forma de protección internacional; $b$ ) la advertencia contra sus connotaciones occidentales e imperialistas; $c$ ) la pretensión de una redefinición minimalista de su catálogo; d) el cuestionamiento de si el Derecho es necesario para asegurar su protección y ejecución, y e) el rechazo de sus declaraciones jurídicas internacionales y de su protección judicial como principales medios que llevan a su violación.

Palabras clave: Ferrajoli, Principia iuris, teoría del Derecho y de la democracia, derechos fundamentales.

ABSTRACT. Principia iuris by L. FERRAJOLI provides a comprehensive theory of law and democracy of singular relevance in the international literature of the last decades. After mentioning some of its primary distinguishing features, this paper focuses on the import of the theoretical and argumentative apparatus of Principia iuris which allows those who cares for their protection to react and reply to the everyday progressive delegitimation of the doctrine and culture of fundamental rights. Namely, it focuses on the import of FERRAJOLI's theory in answering five widespread sort of contentions undermining the doctrine and the culture of fundamental rights: a) the restatement of war as a means for their international protection, $b$ ) the warning against their Western connotation and imperialistic intent; $c$ ) the claim for a minimalist redefinition of their catalogue; $d$ ) the query as to whether law can be taken to be a necessary condition to secure their protection and enforcement, $e)$ the refusal of their (inter)national legal statement and judicial enforcement as the principal means of their violation.

Keywords: FerRajoli, Principia luris, theory of law and democracy, fundamental rights.

\footnotetext{
* Fecha de recepción: 1 de diciembre de 2008. Fecha de aceptación: 18 de diciembre de 2008. trabajo.

*** Quiero agradecer a P. PAROLARI sus comentarios, atentos y precisos, a un primer borrador de este
} 


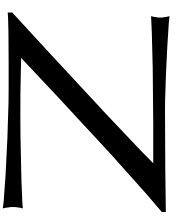

on muchas las razones que hacen de Principia iuris, de L. FERRAJOLI, una obra atípica y singular, una obra rara en la literatura internacional en los últimos decenios. Haré un rápido inventario de las razones que hacen singular la obra $(\$ 1)$, para después detenerme en la que hace de ella un repertorio de argumentos irrenunciables y, antes que eso y aún más significativamente, un sólido aparato conceptual para quien se proponga abordar y hacer frente a la galopante retórica de la antiretórica sobre los derechos fundamentales; esto es, para quien quiera responder a los autores, cada vez más numerosos, que contribuyen a la progresiva deslegitimación de la doctrina y de la cultura de los derechos fundamentales con sus críticas, perplejidades y propuestas reduccionistas o deflacionistas $(\$ 2)$.

\section{UNA OBRA ATIPICA}

Aunque sin pretensiones de completud, y sin profundizar adecuadamente en los diversos temas que se mencionarán, lo que sigue es un reconocimiento sumario de algunas de las razones que hacen singular Principia iuris, desde los puntos de vista metodológico y teórico, y que durante los años de su largo proceso de elaboración han suscitado sobre ella atención e interés, antes incluso de su publicación.

\subsection{Democracia, Derecho y razón}

La primera singularidad y la más manifiesta es el tamaño, que atemoriza y sorprende por igual; un tamaño que, como ha señalado M. ATIENZA, recuerda las grandes obras filosóficas del pasado: imponentes, sorprendentemente rigurosas en su articulación, atentas y cuidadosas en la argumentación. En la mejor tradición de las grandes obras filosóficas, Principia iuris tiene la impronta de una idea fuerte que la funda y que, en una sola frase, sintetiza y deja clara su razón de ser; una frase que pronuncia FERRAJOLI en Camerino, ya diez años antes de la publicación de Principia iuris, en 1997, con ocasión de la concesión de un doctorado honoris causa a N. BOBBIO, entre las conclusiones de su laudatio: «hay una cosa que la historia (del siglo Xx) nos ha enseñado: que en la construcción de la democracia no existen alternativas al Derecho y que, en la construcción del Derecho, no existen alternativas a la razón» ${ }^{1}$.

La convicción de FERRAJOLI en la doble interdependencia entre democracia y Derecho, por una parte, y Derecho y razón, por la otra, ha acompañado toda su producción científica, ya desde la publicación en 1970 de su primer libro, Teoria assiomatizzata del diritto, y aún antes, como él mismo ha tenido ocasión de recordar ${ }^{2}$, desde su tesis de licenciatura, en 1962, Sulla possibilità di una teoria del diritto come scienza rigorosa. Una convicción, la de la doble interdependencia entre democracia y Derecho y entre Derecho y razón, que ha seguido siempre reivindicando y defendiendo con coherente tenacidad en su basta producción científica, en sus numerosas intervenciones en la pren-

\footnotetext{
${ }^{1}$ L. FERRAJOLI, 1999: p. 14

2 L. FERRAJOLI, $2007 \mathrm{~b}$.
} 
sa y en las distintas manifestaciones de su constante dedicación civil, contra toda tentación deconstructivista que tienda hacia el pensamiento débil, y contra toda propensión realista que induzca a la deslegitimación de los principios del Estado de derecho y de los valores de la democracia constitucional; una convicción que en Principia iuris FERRAJOLI ha reafirmado una vez más, delineando una teoría completa y rigurosamente articulada de la misma.

\subsection{Una teoría axiomatizada del iusconstitucionalismo}

Una segunda razón que hace singular la obra, que sorprende aún más que su tamaño, es que en Principia iuris se presenta una teoría axiomatizada del Derecho (del Derecho existente en los países, cada vez más numerosos después de la segunda posguerra, que se han dotado de una constitución escrita, larga, rígida y garantizada); una teoría axiomatizada que se considera funcional y al mismo tiempo complementaria a una teoría de la democracia constitucional (ésta no axiomatizada, pero rigurosamente diseñada y articulada en los diversos elementos que la conforman).

FERRAJOLI procura y defiende decididamente esta singularidad, que no carece de ilustres precedentes en la historia de la filosofía del Derecho (valga por todos la referencia a LEIBNIZ), y no sólo justifica la adopción del método axiomático con «razones teoréticas», como «la simplificación del lenguaje teórico» y «la claridad y univocidad de los conceptos y de las tesis asumidas», sino que también reivindica de esta elección metodológica una «función práctica» porque «el método axiomático representa [...] un potente instrumento de clarificación conceptual, de elaboración sistemática y racional, de análisis crítico y de invención teórica [...] particularmente eficaz para la explicación de la creciente complejidad y falta de efectividad de los ordenamientos modernos, así como también de esquematización de sus modelos normativos y de sus técnicas de garantía» ${ }^{3}$.

En particular, Principia iuris ofrece una teoría axiomatizada del paradigma jurídico que en la literatura iusfilosófica y filosófico-política de los últimos años se ha denominado neoconstitucionalismo y que, quizás para tomar distancia de las versiones del mismo que tienden a sostener una (nueva) versión de cognoscitivismo ético, superponiendo y confundiendo Derecho y moral, FERRAJOLI prefiere denominar «iusconstitucionalismo» ${ }^{4}$. Así pues, desde el punto de vista teórico, Principia iuris ofrece una teoría axiomatizada del paradigma jurídico de los países que, librados de regímenes totalitarios y antiliberales, se han dotado después de la segunda posguerra de una constitución escrita, larga, rígida y garantizada; esto es, una constitución que al posi-

\footnotetext{
3 L. FERRAJOLI, 2007a: vol. 1, pp. vi-vii.

${ }^{4}$ La literatura iusnaturalista del neoconstitucionalismo está tan difundida y enraizada que, para rescatar el modelo teórico de FERRAJOLI de las críticas o, según los casos, de los elogios por sus pretendidas conmistiones con el derecho natural, L. PRIETO, 2008, propone un largo y detallado análisis para mostrar que la que se desarrolla en Principia iuris es «una teoría del Derecho no (neo)costitucionalista para el Estado constitucional». Ahora bien, aunque difundida y enraizada, la teoría de matriz iusnaturalista no es, sin embargo, como presupone también la lectura de PRIETO, la única lectura posible del neoconstitucionalismo, ni la más convincente. Una lectura iuspositivista del neoconstitucionalismo, o, en la terminología de GUASTINI, de la «constitucionalización del ordenamiento», es defendida, por ejemplo, por E. BULYGIN, 2007: p. 186; L. FERRAJOLI, 2006: pp. 23-38; R. GUASINI, 2002, y T. MAZZARESE, 2002a, 2002b y 2007.
} 
tivizar los Derechos fundamentales y el principio del mantenimiento de la paz asume y delimita lo que FERRAJOLI denomina «esfera de lo indecidible»; una esfera de lo indecidible «que y que no», que se sustrae, al menos idealmente, aunque no siempre de hecho, a la discrecionalidad del legislador y se protege de las ingerencias e interferencias del mercado y de la política. En particular, en la caracterización que propone FERRAJOLI, «la esfera del "indecidible que", esto es, de lo que no está permitido (o está prohibido) decidir (está) determinada por el conjunto de los derechos individuales que impiden, como expectativas negativas, decisiones que puedan lesionarlos o reducirlos; y la esfera de lo "indecidible que no", esto es, de lo que no está permitido que no (o es obligatorio) decidir, (está) determinada por el conjunto de los derechos sociales, que imponen, en cuanto expectativas positivas, decisiones idóneas para satisfacerlos» ${ }^{5}$.

\subsection{Un modelo integrado de ciencia jurídica}

Una tercera razón de la singularidad de la obra, por ser poco habitual en la literatura de los últimos decenios, es que Principia iuris, como se ha mencionado anteriormente en relación a sus aspectos metodológicos y teóricos, pretende ofrecer una teoría unitaria y completa en la que el Derecho y la democracia son dos dimensiones complementarias de un mismo esquema conceptual y de un mismo proyecto político ${ }^{6}$; una teoría detallada en su desarrollo y de sorprendente riqueza en la multiplicidad de los temas afrontados. En particular, una teoría unitaria y completa que, como ha subrayado el propio FERRAJOLI, en neta contraposición con una situación «caracterizada por un sustancial analfabetismo jurídico de los filósofos y de un no menor analfabetismo filosófico de los juristas», propone un «modelo integrado de ciencia jurídica» que, sin ignorar las respectivas competencias disciplinares, temáticas y metodológicas, reivindica la complementariedad recíproca entre teoría del Derecho, dogmática, filosofía política y sociología del Derecho ${ }^{7}$.

\subsection{Una irreductible confianza en el diálogo}

Por último, pero no por ello menos importante, otra razón de la singularidad de la obra es que, ya desde años antes de su publicación, Principia iuris está en el centro de un vivaz debate internacional. Durante los cuarenta años que han acompañado su redacción, en efecto, tanto la elección del método axiomático como las distintas tesis en las que FERRAJOLI iba desarrollando y articulando su propia teoría del Derecho y de la democracia constitucional, han sido muchas veces discutidas y sometidas a un cuidadoso examen crítico $^{8}$. FERRAJOLI ha respondido a cada observación, comentario o crí-

L. FERRAJOLI, 2007a: vol. 2, p. 19.

${ }^{6}$ En el mismo sentido se pronuncia G. PRETEROsSI, 2008, quien, entre la literatura de los últimos decenios, considera que, a parte de Principia iuris, la única excepción se encuentra en J. HABERMAS, 1992.

7 L. FERRAJOLI, 2007b.

${ }^{8}$ En particular, son testimonio de este análisis el volumen editado por L. GIANFORMAGGIO en 1993 sobre las tesis y los argumentos de Diritto e ragione; el volumen editado por E. VITALE en 2001, que reproduce (parcialmente) el debate que se desarrolló en las páginas de «Teoría Política» entre 1998 y 2000 sobre los derechos 
tica, testimoniando así su irreductible fe en el diálogo y en la discusión, de forma atenta y puntual, y ésta es una singularidad adicional de Principia iuris, explicando y argumentando en cada ocasión el porqué de sus propias tesis y las razones de los argumentos elegidos para justificarlas. Principia iuris es, en efecto, el resultado de un proceso de redacción y revisión desarrollado ininterrumpidamente durante más de cuarenta años. Un proceso al que ni siquiera la publicación parece haber puesto definitivamente fin, como certifican, en este número de Doxa que publica sus actas, las réplicas de FERRAJOLI, siempre meticulosamente detalladas, a las diversas intervenciones realizadas en el primer seminario dedicado a Principia iuris pocas semanas después de su publicación.

La irreductible fe de FERRAJOLI en el diálogo y la discusión, evoca, casi de forma paradigmática, las tesis habermasianas sobre la acción comunicativa y sobre la teoría discursiva del Derecho y de la democracia; es más, FERRAJOLI parece ir más allá de esas tesis cuando, sin ignorar ni infravalorar las dificultades (teóricas y metateóricas) que pueden prejuzgar los resultados de la acción comunicativa, en abierta contraposición al «pesimismo realista [...], derrotista y paralizante», invita a asumir como «principio metodológico de la acción política y, antes aún, de la filosofía política [...] el optimismo, en cuanto perspectiva de la transformación posible» ${ }^{9}$. El optimismo como principio metodológico, no a pesar de, sino, precisamente, debido a las muchas causas que desde hace algunos años marcan la crisis del constitucionalismo.

\section{EL REALISMO DE UNA TEORÍA POLÍTICAMENTE INACTUAL}

A modo de conclusión de la Prefazione, FerRAJOLI anticipa y resume las críticas de «inactualidad» que podrían dirigirse a Principia iuris, respectivamente, en el plano metateórico, teórico y político: desde el punto de vista metateórico porque la aspiración del neopositivismo lógico, que lo connota fuertemente, parece a estas alturas «abandonada por las más recientes orientaciones postracionalistas»; desde el punto de vista teórico, porque el modelo de Derecho ligado «al paradigma constitucional diseñado por las constituciones de la segunda posguerra» está «cada vez más alejado de las carencias efectivas de las viejas soberanías estatales, de la pérdida de unidad y capacidad regulativa del Derecho y de la complejidad y confusión de sus fuentes de producción»; desde el punto de vista político, finalmente, porque «sugiere la hipótesis de una democracia fundada, a nivel de los ordenamientos estatales y del ordenamiento internacional, sobre la garantía de la paz y de los derechos vitales de todos», en años en los que se asiste, en cambio, «al retorno de la guerra como instrumento de dominio y de solución ilusoria de los problemas internacionales, a procesos de radical desregulación a la vez que de inflación legislativa, a la globalización de la economía y de las comunicaciones, pero no del Derecho y de los derechos, al crecimiento exponencial de las desigualdades y de las devastaciones ambientales, a la muerte cada año de millones de seres humanos por

fundamentales; el volumen sobre el garantismo editado por M. CARBONELL y P. SALAZAR en 2005 y, un año después, la réplica de FERRAJOLI.

${ }^{9}$ L. FERRAJOLI, 2007a: vol. 2, p. 611. 
hambre o por enfermedades no tratadas, a la proliferación de poderes extralegales y salvajes tanto públicos como privados» ${ }^{10}$.

En particular, la inactualidad que FERRAJOLI prevé que desde el punto de vista político se puede objetar a Principia iuris parece ser la de haber puesto a punto un aparato teórico-conceptual en el que la conjugación de Derecho y de democracia constitucional ofrece, finalmente, una teoría unitaria y completa, rigurosamente articulada en sus distintos pasos y sorprendentemente rica en sus contenidos, de la que en 1990 BOBBIO proponía denominar edad de los Derechos, y de la que sintetizaba el núcleo último y fundante afirmando que «[d]erechos del hombre, democracia y paz son tres momentos necesarios del mismo movimiento histórico: sin reconocimiento y protección de los Derechos del hombre no hay democracia; sin democracia no se dan las condiciones mínimas para la solución pacífica de los conflictos» ${ }^{11}$.

Pero, desde el punto de vista político, ¿es realmente inactual, hoy, una teoría que, como la de Principia iuris, conjugue Derecho y democracia constitucional, esto es, que sugiera «la hipótesis de una democracia fundada, al nivel de los ordenamientos estatales y del ordenamiento internacional, sobre la garantía de la paz y de los Derechos vitales de todos»? Y en caso afirmativo, ¿en qué sentido?

Obviamente, si por inactual se entiende minoritaria y a contracorriente respecto de las que, desde hace algunos años, accidental y contingentemente debido al actual (des)orden internacional, constituyen, por un lado, la convicción prevaleciente entre los estudiosos de distintas disciplinas y, por el otro, una práctica recurrente de las políticas de las administraciones de muchos países (occidentales y no occidentales) que contribuyen, la segunda más que la primera, a la progresiva deslegitimación de la doctrina y de la cultura de los Derechos fundamentales, entonces, desde el punto de vista político, la teoría sostenida en Principia iuris es decididamente inactual.

Es más, si por inactual se entiende la difundida falta de efectividad y la reiterada violación de los principios de la doctrina y de la cultura de los Derechos fundamentales, entonces, una vez más, la teoría de Principia iuris es inactual porque, como ha denunciado en diversas ocasiones el propio FERRAJOLI, la edad de los derechos «es también la edad de su violación masiva y de la más profunda e intolerable desigualdad» ${ }^{12}$.

Pero, ¿'son realmente éstos, desde el punto de vista político, los criterios con los que valorar y decidir la actualidad de una teoría del Derecho y de la democracia? ¿Son realmente el parámetro del eventual consenso sobre sus tesis o de la eventual ineficacia de sus principios? Muchos realistas, deconstruccionistas y críticos del Derecho están fuertemente convencidos de ello. Sin embargo, a pesar de su firme convicción, ninguno de los dos criterios es particularmente atendible. No lo es el primero, el criterio del consenso, porque de por sí no proporciona protección alguna contra los riesgos de una dictadura de la mayoría ni contra sus posibles derivas totalitarias. No lo es tampoco el

${ }^{10}$ L. FerRajoli, 2007a: vol. 1, p. ix.

${ }^{11}$ N. BOBBIO, 1990b: p. vii.

${ }^{12}$ L. Ferrajoli, 2004: p. 347. Otros filósofos del Derecho y de la política han realizado observaciones similares, aunque no siempre, como es el caso de FERRAJOLI, para exigir una realización coherente y eficaz de los principios de la edad de los derechos sino más bien, como se señala más adelante $(\$ 2.5)$ respecto del movimiento de los critical legal studies, para deslegitimar la cultura de los que son expresión. 
segundo, el criterio de la ineficacia, porque incurre en lo que los filósofos condenan como falacia naturalista: esto es, la inferencia de una conclusión normativa (la no obligatoriedad o injusticia de un principio) a partir de un dato empírico (su falta de efectividad o su violación).

Ahora bien, si el criterio para decidir acerca de la actualidad de una teoría no es el consenso del que eventualmente gocen sus tesis, ni el grado de efectividad de sus principios, porque ni el uno ni el otro permiten confutar las razones que la justifican y la fundan, entonces, desde el punto de vista político, la teoría propuesta en Principia iuris puede ser considerada una teoría más que nunca actual para quien quiera defender y reafirmar las buenas razones de la doctrina y de la cultura de los Derechos. Una teoría más actual que nunca porque, como se ha señalado ya, no sólo identifica y formula con claridad los argumentos que permiten responder a las siempre más frecuentes reservas, objeciones y críticas que contribuyen a la progresiva deslegitimación de la doctrina y de la cultura de los Derechos, sino sobretodo porque elabora un modelo jurídico y político conjunto (para FERRAJOLI no hay, en efecto, democracia sin Derecho), en el que se configuran los instrumentos para llevar a cabo y garantizar la tutela de los derechos y el mantenimiento de la paz; esto es, un modelo jurídico y político que muestra cómo la tutela de los Derechos y el mantenimiento de la paz no sólo pueden cumplirse sino que, a la larga, resultan una elección más realista y económica que su violación.

En particular, Principia iuris presenta un aparato teórico-conceptual y una amplia gama de argumentos que permiten demostrar que no es convincente ni mucho menos adiáforamente neutra, como a veces se pretende, ninguna de las cinco principales posiciones a las que, quizás simplificando, es posible reconducir muchas críticas de las que ha sido objetivo la doctrina y la cultura de los Derechos, cada vez más a menudo en los últimos años ${ }^{13}$.

\subsection{Las insidias de la «neolengua» de los derechos en la posguerra fría}

El rigor y la claridad con que Principia iuris identifica y articula los principios del Estado de derecho, conjugándolos a los valores de la democracia constitucional, proporcionan, ante todo, un instrumento precioso para desmontar la que es, quizás, la crítica más insidiosa; esto es, la crítica que tiende a deslegitimar la cultura de los derechos, no mediante la puesta en cuestión de sus principios y valores sino, por el contrario, reivindicando la pretensión de quererlos difundir y tutelar. Se trata en particular de la posición de quien, con una destreza lingüística no inferior a la de George Orwell (quien además la había inventado en 1984), repropone una «neo-lengua» en la cual: «la guerra es paz; la libertad es esclavitud; la ignorancia es fuerza»; y, con indudable fantasía onomástica, enriquece su vocabulario siempre con nuevos oxímoron que denominan «inteligentes» las bombas, «vulgar» el pacifismo y «humanitarias» las guerras.

13 Sin hacer referencia a los argumentos propuestos en Principia iuris, la incoherencia y las connotaciones ideológicas de muchas de las críticas a la doctrina y a la cultura de los derechos fueron ya señaladas en T. MAZZARESE, 2006b. 
Pero no sólo esto; se trata también de la posición de quien acompaña esta irrefrenable tendencia a la invención continua de nuevos oxímoron con una notable propensión a los paralogismos y, según los casos, no tiene reserva alguna para afirmar la necesidad de: bombardear con bombas de fragmentación (que luego permanecen durante años en el terreno como minas listas a explotar a cualquier contacto) los lugares en los se lamenta que a los niños les esté prohibido jugar corriendo felices tras una cometa $^{14}$; además de bombardear grandes ciudades y pequeñas poblaciones, casas y mercados donde se encuentran las mujeres a las que se pretende liberar del burka y de la opresión de una cultura integrista que mortifica su dignidad; de contaminar cauces de agua y destruir calles, puentes y toda infraestructura económica de pueblos a los que se quiere liberar de la plaga de la miseria; de profanar y hasta destruir lugares de culto de quienes son acusados de ser integristas e intolerantes hacia otras culturas y religiones ${ }^{15}$; de utilizar armas prohibidas por las convenciones internacionales (frecuentemente ni siquiera ratificadas) y de reproponer incluso el recurso a armas atómicas para evitar el peligro de que otros puedan utilizar o aún sólo disponer de armas de destrucción masiva; de justificar la institución de tribunales militares y cárceles especiales, cuando no incluso el recurso a prácticas como la tortura ${ }^{16}$, en aquellos países de los que se estigmatiza la barbarie de los regímenes totalitarios que los han gobernado.

Esta posición que tiende a deslegitimar la cultura de los derechos, no contestándola sino reinventando su gramática y las modalidades de su declaración, es particularmente insidiosa porque, no obstante la tosquedad de sus argumentos y de su lenguaje, logra frecuentemente distraer la atención de los intereses económicos y de las lógicas de poder de las que es expresión, ofreciendo, a quien quiera cuestionar tanto unos como las otras, el falso blanco de la tutela internacional de los derechos. Por ello, resulta de obvia importancia una teoría que, como la delineada en Principia iuris, permite poner de nuevo orden en el léxico y en el aparato conceptual de los derechos y de sus garantías.

14 Se hace referencia a la guerra en Afganistan, iniciada el 7 de octubre de 2001 en respuesta al ataque terrorista del 11 de septiembre y, con el pasar de los meses, vio justificada su continuación recurriendo (también) a otras y distintas motivaciones como la reivindicación del derecho de los niños a jugar con las cometas o la denuncia de la opresión de las mujeres y del carácter totalitario y antiliberal del régimen talibán.

15 Se hace referencia al bombardeo de la mezquita de Falluja en la primavera de 2004 y a los repetidos combates que se desarrollaron en sus muros.

16 Por lo que respecta a la tortura, es ya conocido que no se ha tratado de casos aislados y no autorizados, como las fuentes oficiales sostuvieron después de las primeras denuncias relativas a la base de Guantánamo en Cuba, a la cárcel de Abu Ghraib en Iraq y a los centros de detención de Bagram y Gardez en Afganistán. Por un lado, en efecto, desde hace ya algunos años los términos de la relegitimación de la tortura han sido objeto de un debate público que, como ha señalado A. M. DerSHOWITZ, 2002: p. 134, tuvo inicio después del 11 de septiembre cuando «FBI agents began to leak stories suggesting that they might have to resort to torture to get some detainees, who were suspected of complicity in Al-Qaeda terrorism, to provide information necessary to prevent a recurrence»; un debate público al que no ha faltado siquiera la contribución de quien, como M. IGNATIEFF, 2004: pp. 136-144, en casos excepcionales como el del terrorismo internacional, ha sugerido una posible justificación del recurso a la tortura en nombre de una «ética del mal menor». Y, por otro lado, junto al debate público sobre los términos teórico-filosóficos de su relegitimación, por parte gubernamental no han faltado admisiones y confirmaciones cada vez más numerosas, explícitas y autoritativas, hasta llegar el 8 de marzo de 2008 al veto opuesto por el Presidente de los Estados Unidos, G. W. Bush, a la ley aprobada por el Congreso para abandonar técnicas de interrogatorio particularmente violentas como la del waterboarding (una suerte de simulación de ahogo). 


\subsection{Universalismo de los derechos y valoración de las diferencias}

Una segunda posición a la que es posible reconducir algunas de las críticas que contribuyen a la deslegitimación de la cultura de los derechos es la de quien denuncia su supuesto imperialismo ético-cultural. A esta posición pueden reconducirse las críticas (heterogéneas en la pluralidad de sus diversas formulaciones y connotaciones) de quien, no sólo en nombre del comunitarismo y del multiculturalismo sino también de la gender theory y más recientemente también de la critical race theory ${ }^{17}$, cuestiona el universalismo de los derechos y reivindica una declaración del catálogo de los derechos que, según los casos, tome en cuenta las diversas identidades culturales y/o de género y/o de «raza» ${ }^{18}$.

Ahora bien, pese a la variedad de acentos en su formulaciones, las críticas al imperialismo ético-cultural de la doctrina de los derechos parecen desconocer la participación de países occidentales y orientales, del norte y del sur del mundo, en la redacción de su catálogo en la Declaración universal, y, sobre todo, parecen no tomar en cuenta que la doctrina de los derechos, más que ninguna otra, está orientada laicamente al respeto del pluralismo cultural y moral. Esto es, esas críticas parecen ignorar, como escribe CASSESE, la conclusión de una detallada reconstrucción crítica de su génesis, que «[1]a Declaración Universal es fruto de varias ideologias: el punto de encuentro y de reunión de concepciones diversas del hombre y de la sociedad» y que «ella no constituye el simple "agrandamiento", a nivel mundial, de textos nacionales, sino la "adaptación" de aquellos textos a un mundo multicultural, profundamente heterogéneo y dividido» ${ }^{19}$.

Es más, siempre en la variedad de acentos de sus distintas formulaciones, las reivindicaciones de una declinación distinta del catálogo de los derechos que tome en consideración y dé cuenta de la especificidad de identidades distintas parece ignorar, como FERRAJOLI ha subrayado en muchas ocasiones, y como repite una vez más, que «el universalismo de los derechos fundamentales [...] no sólo es compatible con el respeto de las diferencias reivindicado por el multiculturalismo, sino que constituye su principal garantía», porque son «los derechos fundamentales, y específicamente los derechos de libertad los que garantizan [...] el valor igual de todas las diferencias personales, empezando por las culturales, que hacen de cada persona un individuo diferente de todos los demás y de cada individuo una persona igual a todas las demás ${ }^{20}$. Esto es, las reivindicaciones de una declinación distinta del catálogo de los derechos fundamentales en función de las distintas identidades parecen ignorar, como confir-

${ }_{17}$ Sobre la critical race theory, aún poco debatida en Europa, cfr., por ejemplo, C. WEST, 1994; K. CRENShaw, N. Gotanda, G. Peller y K. Thomas (eds.), 1995; K. Thomas y G. Zanetti (eds.), 2005, y Th. CasaDEI y L. RE (eds.), 2007.

${ }_{18}$ Aunque en términos bastante problemáticos, manifiesta una posición crítica respecto del universalismo de los derechos, R. Rossanda, 2007, cuando, en la propia recensión a Principia iuris, a pesar de declarar que está «de su parte», formula a FERRAJOLI la pregunta tantas veces planteada por las teóricas de la diferencia de género: «¿es posible que un código, sus lemas y sus jerarquías, escritos por uno de los dos sexos, valga absolutamente para el otro y (que se trate) sólo de aplicarlo con la coherencia que hasta ahora no habría tenido?».

${ }_{19}$ A. CASSESE, 1988, reed. 1999: p. 47, la cursiva es del autor. Con tonos y acentos no siempre coincidentes, un análisis parecido puede encontrarse también, por ejemplo, en M. SALVATI, 2006: p. 34, y en M. FLORES, 2006: pp. 101-104.

${ }^{20}$ L. Ferrajoli, 2007a: vol. 2, pp. 58-59. 
man en cambio las críticas dirigidas en diciembre de 2007 a la ONU y a sus valores por el Papa Benedicto XVI ${ }^{21}$, que la de los derechos es la doctrina que, más que ninguna otra, tiene la impronta laica del respeto del pluralismo cultural y moral. Dicho de otro modo, esas reivindicaciones parecen ignorar, como FERRAJOLI exhorta en cambio a no olvidar, que «la primera libertad afirmada en Europa ha sido la libertad de conciencia, que es un típico derecho cultural, es más, el primer y fundamental derecho a la tutela de la propia identidad y diferencia cultural», y que la libertad religiosa, la libertad de manifestación del pensamiento y otras libertades fundamentales «sirven todas para tutelar la identidad diversa, disidente, no homologable de cada persona» ${ }^{22}$.

\subsection{El aparente pragmatismo antiretórico del minimalismo de los derechos}

Entre las distintas formas de deslegitimación de la doctrina y de la cultura de los derechos, en los últimos años ha obtenido una creciente atención y siempre mayores consensos la del denominado minimalismo. La propuesta de poner en marcha una política deflacionista de los derechos, expresión aparente de un pragmatismo supuestamente no condicionado por compromisos ideológicos, se presenta, en efecto, como la única solución razonablemente practicable para contener la crisis de la edad de los derechos. Cautivante en la sobriedad de su formulación, la solución indicada por el minimalismo prevé una doble estrategia: ante todo una cuidadosa selección, que quizás podría ser sancionada en una «Segunda Declaración Universal» ${ }^{23}$, de los derechos «realmente» esenciales entre aquellos que se confunden y se superponen en una inflación ya fuera de control y, contextualmente, una adecuada redefinición de los mecanismos que permitan asegurar una tutela «realmente» eficaz de los derechos «realmente» esenciales. A pesar de la referencia al pragmatismo y la intolerancia declarada a la retórica de «un humanismo que se adora a sí mismo» ${ }^{24}$, la del minimalismo no es —como

${ }^{21}$ Se hace referencia al discurso que Benedicto XVI realizó el 1 de diciembre de 2007 a las organizaciones no gubernamentales de inspiración católica; en ese discurso, Benedicto XVI deploró, en efecto, que «a menudo, el debate internacional (se presente) marcado por una lógica relativista que parece sostener, como única garantía de una convivencia pacífica entre los pueblos, la negación de ciudadanía a la verdad sobre el hombre y sobre su dignidad, así como también a la posibilidad de un actuar ético fundamentado en el reconocimiento de la ley moral natural». Es más, algunos meses después, a pesar del tono aparentemente más conciliador, Benedicto XVI manifestó de nuevo su preocupación por la posible deriva relativista de los derechos fundamentales, en New York, en el discurso ante la Asamblea de las Naciones Unidas del 18 de abril de 2008. En esta ocasión, en efecto, Benedicto XVI reconoció que la declaración universal de 1948 «fue el resultado de una convergencia de tradiciones religiosas y culturales, todas ellas motivadas por el deseo común de poner a la persona humana en el corazón de las instituciones y leyes y acciones sociales», pero, una vez más, ha destacado especialmente el peligro de «ceder a una concepción relativista, para la que el significado y la interpretación de los derechos podrían variar y su universalidad (ser) negada en nombre de contextos culturales, políticos, sociales e incluso religiosos diferentes».

${ }^{22}$ L. FERRAJOLI, 2007a: vol. 2, p. 59.

23 Así, por ejemplo, A. FERRARA, 2003a, escribe que una «Segunda Declaración Universal de los derechos Humanos», oportunamente circunscrita a «aquellos poquísimos derechos genuinamente fundamentales», podría «con indiscutida autoridad jurídica y no sólo moral, identificar con claridad [...] qué tipos de violación de los derechos humanos fundamentales constituyen motivo para una legítima intervención militar que sólo entonces podría despojarse del título de "guerra” y asumir el de "operación de policía”»; cfr., además, A. FERRARA, 2003b: pp. 398-399.

${ }^{24}$ Referencia a M. IgNATIEFF, 2001b: p. 53. 
pretende y reivindica - una «razonable apología de los derechos» ${ }^{25}$ sino, por el contrario, una forma radical de deslegitimación de su cultura. En efecto, independientemente de cualquier otra referencia a la (in)coherencia y el compromiso ideológico de los argumentos en sus diversas formulaciones ${ }^{26}$, resulta manifiesto que el minimalismo no se preocupa tanto de eliminar las reivindicaciones excéntricas y las pretensiones risibles de su catálogo, sino más bien de negar la indivisibilidad de los derechos políticos y sociales, civiles y económicos que caracterizaron su afirmación desde la Declaración universal de 1948. De manera no menos abierta el minimalismo cuestiona sobre todo la formulación pacifista de la Carta de la ONU, en nombre de intervenciones que permitan una eficaz oposición a las violaciones más graves de los derechos considerados esenciales, y solicita su reforma en el sentido de una reedición de la doctrina de la guerra justa que, siguiendo las tradiciones más sobresalientes, codifique las condiciones de su legitimidad ${ }^{27}$.

Son al menos tres los aspectos de la propuesta de una política deflacionista de los derechos fundamentales que las tesis de Principia iuris y los argumentos que las justifican y las fundamentan contribuyen a refutar: i) el primero es el que identifica en una presunta atención por el multiculturalismo la razón y la necesidad de una redefinición minimalista del catálogo de los derechos; ii) el segundo es el relativo al «costo de los derechos», considerado un obstáculo especialmente por la tutela de los derechos sociales, y iii) el tercero, poco coherente con la declarada preocupación por el costo de los derechos, así como con la presunta atención por los valores de las otras culturas, distintas de la «cultura occidental», es el de la relegitimación de la guerra replanteado como instrumento al que recurrir allá donde se esté en presencia de una (no mejor definida) gross violation de los «verdaderos» derechos fundamentales identificados por la redefinición de su catálogo.

Ya se ha hecho referencia $(\$ 2.2)$ a las tesis y a los argumentos de Principia iuris para contestar a la presunta tensión dialéctica entre multiculturalismo y tutela de los derechos fundamentales.

De su importancia, en cambio, para contestar a la relegitimación de la guerra, propuesta en algunas de las versiones del minimalismo de los derechos, es difícil decir algo en pocas palabras; en Principia iuris, en efecto, el valor del mantenimiento de la paz es absolutamente central, tanto en el plano de la teoría del derecho como en el de la teoría de la democracia constitucional; es tan central, que no prevé ninguna excepción, más que la enunciada en el art. 51 de la Carta de la ONU de 1945, es decir: «el derecho inmanente de legítima defensa, individual o colectiva, en caso de ataque armado contra un miembro de las Naciones Unidas, hasta que el Consejo de Seguridad haya tomado las medidas necesarias para mantener la paz y la seguridad internacionales»; ex-

\footnotetext{
25 «Una razonable apología de los derechos humanos» es el título elegido para el volumen que reúne la traducción italiana de M. IGNATIEFF, 2001b y 2001c.

${ }^{26}$ Una de las versiones del minimalismo que ha suscitado gran interés es la de M. IGNATIEFF, 2001a, 2001b y 2001c, pero, también entre las reformulaciones que se remiten a ella, no faltan diferencias relevantes. Véanse, por ejemplo, las propuestas por A. FERrARA, 2003a, S. VECA, 2003, G. PRETEROSSI, 2004, y A. CASSESE, 2005. Para una crítica del minimalismo de los derechos, también, aunque no sólo, en la versión propuesta por IGNATIEFF, $c f r$. T. MAZZARESE, 2006a.

${ }_{27}$ Así, por ejemplo, aunque con argumentos y acentos diversos, no sólo M. IGNATIEFF, 2001a, 2001b y 2001c, sino también A. Cassese, 2005, A. Ferrara, 2003a, G. Preterossi, 2004, M. Walzer, 2006.
} 
cepción ésta, que, según escribe FERRAJOLI, en rigor no es calificable como «guerra» sino más bien como «legítima defensa de la guerra» ${ }^{28}$.

Finalmente, respecto del segundo aspecto, en Principia iuris FERRAJOLI no niega en absoluto que los derechos fundamentales conlleven un coste, y que no sólo cuestan «los derechos sociales y la democracia social», sino también los derechos individuales, desde los derechos de libertad hasta los derechos de autonomía, que «incluyen expectativas positivas y requieren, por tanto, un "hacer" de la esfera pública ${ }^{29}$. Sin embargo, para FERRAJOLI, «la tesis de que el costo de los derechos haría imposible su plena satisfacción» es un non sequitur ${ }^{30}$. No sólo porque «las instituciones políticas, empezando por el Estado, no son sociedades mercantiles con finalidad de lucro» que tienen en el crecimiento económico y en la producción de riqueza sus propias finalidades y parámetros de legitimidad, sino sobre todo porque «si es verdad que los derechos fundamentales cuestan, lo es también que cuestan mucho más sus violaciones y sus incumplimientos» ${ }^{31}$. Y que la violación de los derechos tiene un costo superior al de su ejecución y tutela es una tesis que FERRAJOLI defiende en Principia iuris, reivindicando como necesaria para el desarrollo económico y el crecimiento del bienestar no sólo, como sostiene A. SEN, la garantía de los derechos de libertad, sino también la de los derechos sociales.

\subsection{Reivindicación de la naturaleza moral de los derechos y escepticismo sobre su tutela jurídica}

Una posición distinta que contribuye también, a veces con independencia de las intenciones de quien la sostiene, a la deslegitimación de la cultura de los derechos, se caracteriza no por un cautivador pragmatismo, como en el caso del recién mencionado minimalismo, sino más bien por una suerte de escepticismo respecto del Derecho (positivo). Ésta es, más precisamente, la posición de quien, convencido de su naturaleza moral $^{32}$, objeta la importancia de la positivización de los derechos fundamentales y de

${ }^{28}$ L. FERRAJOLI, 2007a: vol. 2, p. 502.

29 L. FerRajoli, 2007a: vol. 2, p. 67.

${ }^{30}$ Perentoria en su formulación, esta réplica de FERRAJOLI no responde tanto, en realidad, a la tesis del «coste de los derechos», tal como ha sido formulada en el afortunado ensayo de S. HoLmES y C. S. SUNSTEIN, 1999, sino más bien a las diversas reformulaciones que se han propuesto de esta tesis por parte de quienes la han utilizado como argumento para negar la ejecutabilidad y la tutela de los derechos sociales y económicos. En particular, según S. HOLMES y C. S. SUNSTEIN, 1999, «those who object to welfare rights because they cost money should not assume that property rights can be fully safeguarded [...] Our freedom from government interference is no less budget-dependent than our entailment to public assistance. Both freedoms must be interpreted. Both are implemented by public officials who, drawing on the public purse, have good deal of discretion in construing and protecting them» (p. 119); pero la constatación de que «all rights are open-ended for the simple reason that rights have costs and hence can never be perfectly or completely protected» (p. 120), plantea, y no elude en absoluto, cuestiones como: «Who decides at what level to fund which cluster of basic rights to whom?», dado que «attention to the costs of rights leads us not only into problem of budgetary calculation [...] but also into basic philosophical issues of distributive justice and democratic accountability» (p. 131).

${ }^{31}$ L. FERRAJOLI, 2007a: vol. 2, p. 68.

32 En efecto, Moreso y POGGE, citados en el texto para ejemplificar esta posición teórica, no objetan tanto la cultura de los derechos como la tesis de quien, como FERRAJOLI, desconoce la presunta naturaleza moral de los derechos fundamentales. Y, precisamente, para reivindicar su naturaleza moral, uno y otro ponen en discusión, sin indicar, no obstante, sus posibles alternativas, la utilidad del instrumento jurídico para su tutela y 
los procedimientos para su ejecución y tutela jurídica, poniendo en duda, en particular, la relevancia de un mecanismo de control jurisdiccional de la constitucionalidad, dado que «[h] ay sociedades que no disponen de este mecanismo y los derechos aparecen razonablemente garantizados (Suecia) y hay sociedades que disponen del mecanismo (Perú) y, sin embargo, los derechos no están garantizados» ${ }^{33}$.

Es ésta, por ejemplo, la tesis de quien, como Th. POGGE, considera excesiva, y al mismo tiempo insuficiente, la exigencia de que «each society's government and citizens ought to ensure that all human rights are incorporated into its fundamental legal texts and are, within its jurisdiction, observed and enforced through an effective judicial system» ${ }^{34}$. En particular, según POGGE, esta exigencia es excesiva «because a society may be so situated and organized that its members enjoy secure access to [the buman right] $X$, even without a legal right thereto», y es al mismo tiempo insuficiente «because legal and even constitutional rights, however conscientiously enforced, often do not suffice to ensure secure access» ${ }^{35}$.

Y esta es también la posición de quien, como J. J. MORESO, apelando a la tesis de POGGE, critica lo que denomina como «hiperjuridicismo» del modelo constitucionalista propuesto por FERRAJOLI (también, pero no sólo) en Principia iuris ${ }^{36}$.

Ahora bien, si por un lado es obvio, sino incluso banal, negar que el Derecho sea, o pueda ser, un instrumento suficiente, por el otro resulta, en cambio, al menos arriesgado objetar que el Derecho pueda ser, y sea, un instrumento necesario para la efectiva implementación y tutela de los derechos fundamentales: es banal negar que el Derecho sea, o pueda ser, un instrumento suficiente porque el Derecho es norma y prescripción y no hay obligación o prohibición que no pueda ser desatendida o violada; es, en cambio, arriesgado objetar que el Derecho pueda ser, y sea, un instrumento necesario porque, de ese modo, se deja la efectiva implementación y tutela de los derechos fundamentales a merced de disposiciones morales o propensiones naturales cuya difusión y consenso espontáneos resultan cuanto menos improbables. Bastará algún ejemplo.

En primer lugar, para empezar con un caso relativo a Suecia, a la que hace referencia MORESO, aunque no haya sido en absoluto suficiente, sin la declinación jurídica de sus formas y de sus modos, el welfare de las socialdemocracias escandinavas no se ha-

ejecución. Una posición análoga ha sido sostenida por Benedicto XVI, 2008, afirmando que «cuando son presentados simplemente en términos de legalidad, los derechos corren el riesgo de resultar débiles proposiciones separadas de la dimensión ética y racional, que es su fundamento y finalidad», y enfatiza que «los derechos humanos deben ser respetados como expresiones de la justicia y no simplemente porque pueden ser hechos respetar mediante la voluntad de los legisladores».

${ }^{33}$ J. J. MORESO, 2008. Además de las razones mencionadas en el texto, esta idea de MORESO resulta dudosa y problemática porque parece ignorar que en Suecia no sólo existe un control de constitucionalidad, sino que lo hay incluso por partida doble: existe una primera forma de control, centralizado y ex ante, confiado al Lagrådet (Consejo legislativo), un órgano compuesto por ex jueces de la Corte Suprema y de la Corte Suprema Administrativa, y un segundo tipo de control, difuso y ex post, confiado a los tribunales ordinarios.

${ }^{34}$ Th. PogGe, 2002, reed. 2008: p. 51.

${ }^{35}$ Ibidem.

36 Obsérvese, a modo de inciso, que esta crítica de «hiperjuridicismo» parece desmentir la lectura en clave iusnaturalista que se ha propuesto muchas veces (también por MORESO, aunque no sólo de él) del constitucionalismo de FERRAJOLI; lectura, ésta, a la que FERRAJOLI ha respondido en diversas ocasiones reafirmando los términos iuspositivistas de su propio modelo teórico. 
bría podido llevar a cabo como simple expresión de una natural disposición de ánimo a la solidaridad de las poblaciones nórdicas; del mismo modo que, en el curso de los años, sus diversas vicisitudes no han sido el resultado de ocasionales cambios en los valores de esas poblaciones, sino más bien de las distintas intervenciones legislativas que, en cada momento, han redefinido sus términos.

Tomando ahora un ejemplo del Derecho internacional: aunque en absoluto suficientes para impedir la práctica de la tortura o el uso de armas químicas, la inexistencia de la Convención internacional de 1984, que prohíbe la primera, y la inexistencia de la Convención internacional de 1997, que ha decretado la prohibición de las segundas, habría beneficiado sólo a quien, como en el caso de los centros de detención de Guantánamo y de Abu Ghraib o en el caso de la utilización del fósforo blanco durante el ataque a Fallujah en noviembre de 2004 (fracasado ya todo intento de desmentirlo) no ha encontrado otra justificación a sus propias acciones que la apelación a la falta de ratificación de esas convenciones.

Más aún, siempre en el ámbito del Derecho internacional, es un dato claramente incontestable que la prohibición de la guerra en el Pacto Briand Kellog, en 1928, no consiguió evitar la II Guerra Mundial y que su solemne reafirmación en la Carta de la ONU de 1945 no ha sido suficiente para evitar, durante y después de la guerra fría, la multiplicación de una pluralidad de conflictos regionales y globales. Es, sin embargo, un dato incontestable que, por sí mismo, sólo testimonia la reiterada violación de una norma que, desde la finalización de la I Guerra Mundial, se ha intentado que forme parte de una común cultura de la paz y de la tutela de los derechos fundamentales; esa reiterada violación testimonia indudablemente la insuficiencia de esa norma, pero no su inutilidad, del mismo modo que la reiterada violación de las más elementales normas del Derecho penal son testimonio de su insuficiencia pero no de su inutilidad. Normas, éstas, de las que, con independencia de cuál sea su grado de cumplimiento, difícilmente se discutiría su carácter necesario.

Es innegable, por tanto, como con razón subrayan POGGE y MORESO, que la positivización de los derechos fundamentales, en el Derecho nacional e internacional, no es suficiente por sí misma para su plena y completa implementación, como tampoco lo sería, verosímilmente, la puesta en marcha de todas las instituciones de garantía que integran el rico y detallado elenco propuesto por FERRAJOLI en Principia iuris. Pero, aunque unas y otras puedan no ser suficientes, el Derecho parece ser necesario para su tutela, como advierte H. ARENDT cuando afirma el carácter irrenunciable del «derecho a tener derechos» ${ }^{37}$.

\subsection{No hay alternativa a la protección de los derechos en la defensa de la paz y de la libertad}

Finalmente, una última posición es la de quien no se limita a problematizar las dificultades de carácter político y jurídico que interfieren en el reconocimiento y la tutela de los derechos, sino que, precisamente, sitúa en estas dificultades la razón última y

${ }^{37}$ H. ARENDT, 1951. 
definitiva del fracaso necesario de la edad de los derechos y de sus principios. Esta posición, recurrente entre los seguidores del movimiento de los critical legal studies y del realismo, considera las graves y reiteradas violaciones de los derechos del siglo XX (aunque no sólo de él), un efecto de las diversas declaraciones y convenciones que han sancionado el reconocimiento y la necesidad de su tutela internacional; un efecto y no simplemente una prueba de su ineficacia.

Así, por ejemplo, Costas DouZINAS interpreta como una consecuencia de la naturaleza intrínsecamente «aporética» (de la doctrina) de los derechos ${ }^{38}$ el récord impresionante de sus violaciones «since their ringing declarations at the end of eighteen century» y enfatiza que «[i]f the twentieth century is the epoch of human rights, their triumph is, to say the least, something of a paradox. Our age has witnessed more violations of their principles than any of the previous and less "enlighted" epochs. The twentieth century is the century of massacre, genocide, ethnic cleansing, the age of the Holocaust» ${ }^{39}$. Además, la tesis de que toda versión de la declaración y regulación jurídica de la tutela (inter)nacional de los derechos conlleva su inevitable desnaturalización, desconocimiento y violación es asumida por quien, como M. KOSKENNIEMI, está convencido de que «while the rhetoric of human rights has historically had a positive and liberating effect on societies, once rights become institutionalized as a central part of political and administrative culture, they lose their transformative effect and are petrified into a legislative paradigm that marginalizes values or interests that resist translation into rights-language» ${ }^{40}$.

Ahora bien, aunque existan sin duda motivos para el pesimismo y estén justificadas las reservas acerca de los límites y las posibles contradicciones de su institucionalización, de ello no se infiere que el rechazo del Derecho como instrumento de implementación y de tutela de los derechos pueda resolver, y no más bien replantear (de forma incluso más dramática), el problema de la defensa y de la salvaguardia de los valores expresados por los derechos. En efecto, una vez abandonado el Derecho porque resulta un instrumento quizás demasiado insidioso, no quedaría otra alternativa que un uso indiscriminado de la fuerza y del arbitrio, mucho menos tranquilizador. Así, en conclusión, la que parece intrínsecamente «aporética» no es la doctrina de los derechos, con todas las dificultades que la hacen controvertida, sino más bien la posición de quien decreta su fracaso y auspicia su fin no porque cuestione sus valores sino, sorprendentemente, porque lamenta su violación.

Más allá de la denuncia de su incoherencia intrínseca, no hay réplica más apropiada al pesimismo de esta posición, que enfatiza las graves y reiteradas violaciones de los derechos fundamentales, que el «optimismo metodológico» que FERRAJOLI reivindica en las páginas conclusivas de Principia iuris; un optimismo metodológico que el propio FERRAJOLI sintetiza recurriendo a una cita de BOBBIO, con la que, significativamente, concluye su trabajo: «Respecto de las grandes aspiraciones del hombre estamos ya muy retrasados. Intentemos no aumentar el retraso con nuestra desconfianza, con nuestra indolencia, con nuestro escepticismo. No tenemos tiempo que perder. La historia, como

\footnotetext{
38 C. Douzinas, 2000: p. 21.

39 Ibidem, p. 2.

${ }^{40}$ M. Koskenniemi, 1999: p. 99.
} 
siempre, se mueve hacia dos direcciones opuestas: hacia la paz o la guerra, hacia la libertad o la opresión. El camino de la paz y de la libertad pasa, sin duda, a través del reconocimiento y la protección de los derechos del hombre [...]. No escondo que el camino es difícil. Pero no hay alternativas» ${ }^{41}$.

\section{BIBLIOGRAFÍA}

AREndT, H., 1951: The Origins of Totalitarianism, New York: Harcourt Brace Jovanovich.

BenedeTto XVI, 2007: Discorso alle ong cattoliche, 1 de diciembre de 2007, en http://www.ratzingerbenedettoxvi.com/ongcattoliche.htm.

- 2008: Discorso all'ONU, 18 de abril de 2008, en http://www.ratzingerbenedettoxvi.com/usonu.htm.

BoBBIO, N., 1988: «Dalla priorità dei doveri alla priorità dei diritti», en Mondoperaio, 41, pp. 57 60; reed. en N. BobBIO, 1999: Teoria generale della politica, Torino: Einaudi, pp. 431-440.

— 1990: «Introduzione», en N. BobBio, L'età dei diritti, Torino: Einaudi, pp. 5-16.

Bulygin, E., 2007: «Tecla Mazzarese sobre el positivismo y la globalización del Derecho», en J. J. MoRESO y M. C. REDONDO (eds.), Un diálogo con la teoría del derecho de Eugenio Bulygin. Madrid: Marcial Pons, pp. 185-186.

Carbonell, M., y Salazar, P. (eds.), 2005: Garantismo. Estudios sobre el pensamiento jurídico de L. FERRAJOLI, Madrid: Trotta.

CASADEI, Th., y RE, L. (eds.), 2007: Differenza razziale, discriminazione e razzismo nelle società multiculturali. Reggio Emilia, Diabasis, 2 vols.

CASSESE, A., 1988: «La Dichiarazione universale dei diritti umani. Quaranta anni dopo», en A. CASSESE, I diritti umani nel mondo contemporaneo. Roma-Bari: Laterza, reed. 1999, pp. 2-49.

- 2005: «Ripensare i diritti umani: quali prospettive per i prossimi decenni?», en A. CASSESE, I diritti umani oggi, Roma-Bari: Laterza, pp. 211-229.

Crenshaw, K.; Gotanda, N.; Peller, G., y Thomas, K. (eds.), 1995: Critical Race Theory: The Key Writings that Formed the Movement, New York: New Press.

Dershowitz, A. M, 2002: Why Terrorism Works. Understanding the Threat, Responding to the Challenge, New Haven: Yale University Press.

DouzINAS, C., 2000: The End of Human Rights. Critical Legal Thought at the Turn of the Century, Oxford: Hart.

FERRAJOLI, L., 1970: Teoria assiomatizzata del diritto, Milano: Giuffrè.

- 1989: Diritto e ragione. Teoria del garantismo penale, Roma-Bari: Laterza.

— 1999: «Ragione, diritto e democrazia nel pensiero di Norberto Bobbio», en L. FERRAJOLi y P. DI LuCIA (eds.), Diritto e democrazia nella filosofia di Norberto Bobbio, Torino: Giappichelli, pp. 5-14.

- 2001: Diritti fondamentali. Un dibattito teorico, E. Vitale (ed.), Roma-Bari: Laterza.

- 2004: Diritti fondamentali e democrazia costituzionale, en P. COMANDUCCI y R. GUASTINI (eds.), Analisi e diritto 2002-2003, Torino: Giappichelli, pp. 331-350.

- 2006: Garantismo. Una discusión sobre derecho y democracia, Madrid: Trotta.

- 2007a: Principia iuris. Teoria del diritto e della democrazia, Roma-Bari: Laterza, 3 vols.

- 2007b: Per una rifondazione epistemologica della teoria del diritto, manuscrito.

${ }^{41}$ L. FerRAJOLI, 2007a: vol. 2, p. 612, la cita corresponde a N. BoBBIO, 1988, sec. ed. 1999, pp. 439-440. 
FERRARA, A., 2003a: Fondare senza fondamentalizzare i diritti umani: il ruolo di una seconda Dichiarazione, en http://www.juragentium.unifi.it, Discussioni on line, Forum Ignatieff, Interventi.

— 2003b: «Two Notions of Humanity and the Judgment Argument for Human Rights», en Political Theory, 31, n. ${ }^{\circ}$ 3, pp. 392-420.

FlORES, M., 2006: «Da Norimberga ai diritti umani nel secolo mondo», en M. SALVATI (ed.), Dichiarazione universale dei diritti dell'uomo 10-12-1948: nascita, declino e nuovi sviluppi, Roma: Ediesse, pp. 95-106.

Gianformaggio, L. (ed.), 1993: Le ragioni del garantismo. Discutendo con Luigi Ferrajoli, Torino: Giappichelli.

GuASTINI, R., 2002: «La “costituzionalizzazione” dell'ordinamento», en T. MAZZARESE (ed.), Neocostituzionalismo e tutela (sovra)nazionale dei diritti fondamentali. Torino: Giappichelli, pp. 147-72.

Habermas, J., 1992: Faktizität und Geltung. Beiträge zur Diskurstheorie des Recths und des demokratischen Rechtsstaats, Frankfurt am Main: Suhrkamp.

Holmes, S., y Sunstein, C. R., 1999: The Cost of Rights. Why Liberty Depends on Taxes, New York: W. W. Norton.

IgnatiefF, M., 2001a: «Dignity and Agency», en A. Gutmann (ed.), Human Rights as Politics and Idolatry, Princeton: Princeton University Press, pp. 161-173.

— 2001b: «Human Rights as Idolatry», en A. GuTMANN (ed.), Human Rights as Politics and Idolatry, Princeton: Princeton University Press, pp. 53-98.

— 2001c: «Human Rights as Politics», en A. Gutmann (ed.), Human Rights as Politics and Idolatry, Princeton: Princeton University Press, pp. 3-52.

- 2004: The Lesser Evil: Political Ethics in an Age of Terror, Princeton: Princeton University Press.

Koskenniemi, M., 1999: «The Effect of Rights on Political Culture», en Ph. Alston (ed.), The EU and Human Rights, Oxford: Oxford University Press, pp. 99-116.

MAZZARESE, T., 2002a: «Diritti fondamentali e neocostituzionalismo: un inventario di problemi», en T. MAZZARESE (ed.), Neocostituzionalismo e tutela (sovra)nazionale dei diritti fondamentali, Torino: Giappichelli, pp. 1-69.

- 2002b: «Towards a Positivist Reading of Neo-constitutionalism», en Associations. Journal of Legal and Social Theory, 6, n. ${ }^{\circ}$ 2, pp. 233-60.

- 2006a: «Minimalismo dei diritti: pragmatismo antiretorico o liberalismo individualista?», en Ragion Pratica, n. ${ }^{\circ}$ 26, pp. 179-208.

- 2006b: «¿Repensar la cultura de los derechos?», en Revista internacional de filosofía política, 28, pp. 149-172.

— 2007: «Iuspositivismo y globalización del derecho: ¿Qué modelo teórico?», en J. J. MorESO y M. C. REDONDO (eds.), Un diálogo con la teoría del derecho de Eugenio Bulygin, Madrid: Marcial Pons, pp. 61-71.

Moreso, J. J., 2008: «Ferrajoli o el constitucionalismo optimista», en Doxa, 32, n. ${ }^{\circ} 2$.

Pogge, Th., 2002, reed. 2008: World Poverty and Human Rights, Cambridge: Polity Press.

Preterrossi, G., 2004: L'Occidente contro se stesso, Roma-Bari: Laterza.

- 2008: «Principia iuris tra normatività e potere: sullo stato costituzionale di diritto nella teoria di Luigi Ferrajoli», en Doxa, 32, n. ${ }^{\circ} 2$.

PRIETO SANCHís, L., 2008: «Principia iuris: una teoría del derecho no (neo)constitucionalista para el estado constitucional», en Doxa, 32, n. ${ }^{\circ} 2$.

Rossanda, R., 2007: «La passione del fare politico», en Il manifesto, 5 de diciembre de 2007. 
Salvati, M., 2006: «Alle origini della Carta del 1948», en M. SAlvati (ed.), Dichiarazione universale dei diritti dell'uomo 10-12-1948: nascita, declino e nuovi sviluppi, Roma: Ediesse, pp. 9-38.

Thomas, K., y ZanetTi, G. (eds.), 2005: Legge, razza, diritti. La Critical Race Theory negli Stati Uniti, Reggio Emila: Diabasis.

VECA, S., 2003: «I diritti umani e la priorità del male», en M. IGNATIEFF, Una ragionevole apologia dei diritti umani, Milano: Feltrinelli, pp. 101-134.

Walzer, M., 2006: «Oltre l'intervento umanitario», en Lezioni Bobbio. Sette interventi su etica e politica, Torino: Einaudi, pp. 3-21.

West, C., 1994: Race Matters, New York: Vintage.

(Traducción de Jordi Ferrer Beltrán) 